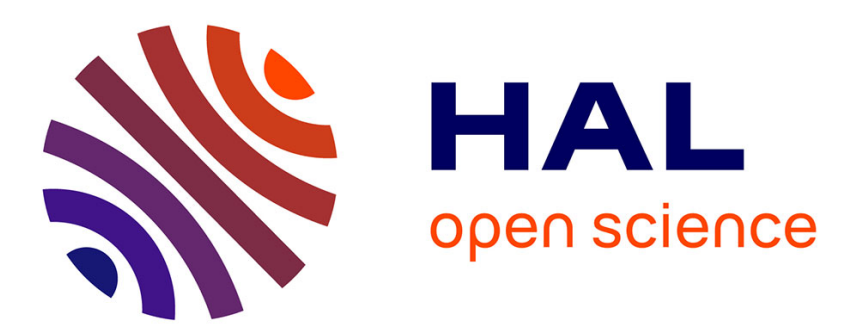

\title{
Kerr optical frequency comb generation using whispering-gallery-mode resonators in the pulsed-pump regime
}

Thomas Daugey, Cyril Billet, John Michaël Dudley, Jean-Marc Merolla, Kouomou Yanne Chembo

\section{To cite this version:}

Thomas Daugey, Cyril Billet, John Michaël Dudley, Jean-Marc Merolla, Kouomou Yanne Chembo. Kerr optical frequency comb generation using whispering-gallery-mode resonators in the pulsed-pump regime. Physical Review A, 2021, 103, pp.023521 (7). hal-03360037

\section{HAL Id: hal-03360037 https://hal.science/hal-03360037}

Submitted on 30 Sep 2021

HAL is a multi-disciplinary open access archive for the deposit and dissemination of scientific research documents, whether they are published or not. The documents may come from teaching and research institutions in France or abroad, or from public or private research centers.
L'archive ouverte pluridisciplinaire HAL, est destinée au dépôt et à la diffusion de documents scientifiques de niveau recherche, publiés ou non, émanant des établissements d'enseignement et de recherche français ou étrangers, des laboratoires publics ou privés. 


\title{
Kerr optical frequency comb generation using whispering-gallery mode resonators in the pulsed-pump regime
}

\author{
Thomas Daugey $^{1}$, Cyril Billet ${ }^{1}$, John Dudley ${ }^{1}$, Jean-Marc Merolla ${ }^{1}$, and Yanne K. Chembo ${ }^{2 *}$ \\ ${ }^{1}$ Institut FEMTO-ST, UMR6174, CNRS Université Bourgogne Franche-Comté, \\ 15B Avenue des Montboucons, 25030 Besançon cedex, France. \\ ${ }^{2}$ University of Maryland, Department of Electrical and Computer Engineering \\ G6 Institute for Research in Electronics and Applied Physics (IREAP), \\ 8279 Paint Branch Dr, College Park MD 20742, USA
}

(Dated: January 24, 2021)

\begin{abstract}
Kerr comb generation is usually based on the nonlinear dynamics of the intracavity field in a whispering-gallery mode resonator pumped by a continuous-wave laser. However, using a pulsed instead of a continuous-wave pump opens a new research avenue from both the theoretical and experimental viewpoints, as it permits to tailor the spectral properties of ultrashort pulse trains with a single passive nonlinear element. In this article, we study the dynamics of Kerr optical frequency combs when the whispering-gallery mode resonator is pumped by a synchronous pulsetrain. We propose a model that is based on an extension of the Lugiato-Lefever equation, which accounts for both the pulsed nature of the pump and the mismatch between the free-spectral range of the resonator and the repetition rate of the pulse train. We lay a particular emphasis on the effect of pump-cavity desynchronization on the spectral shape of the output combs. The numerical simulations are successfully compared with experimental measurements where the optical pulses are generated via time-lens soliton compression, and the resonator is a millimeter-size magnesium fluoride resonator with billion quality factor at the pump wavelength.
\end{abstract}

\section{INTRODUCTION}

Whispering-gallery mode (WGM) resonators with Kerr nonlinearity are a compact and versatile platform for the generation of broadband optical frequency combs [1-4]. The intracavity process is based on stimulated four-wave mixing, and the spectral features of the comb depend in a non-trivial fashion on the intrinsic properties of the cavity (mainly dispersion and losses), and on those of the continuous-wave pump (power and frequency). The main appeal of Kerr comb generation using a continuous-wave pump is its conceptual simplicity and its high conversion efficiency.

A complementary approach to Kerr comb generation corresponds to the case where the resonator is pumped in a synchronous pulsed regime. In the temporal domain, the lightwave signal entering the cavity is an optical pulse train with a repetition rate matching the free-spectral range of the cavity. In the spectral domain, this corresponds to pumping the cavity with an optical frequency comb. The output comb therefore results from the nonlinear interaction of the input comb with the WGM resonator. The resonator therefore responds as if it was subjected to a large number of mutually coherent pumps, and its dynamical behavior becomes substantially different than the one obtained when there is a single-frequency pump. The earliest works along that line, even though not exactly viewed as pulse pumping schemes, involved dual-pump Kerr combs - the "pulses" in this case being mere sinusoids when both modes are phase-locked [5-

\footnotetext{
*ykchembo@umd.edu
}

9]. Recently, more systematic experimental works have shown that pumping high- $Q$ microresonators with ultrashort optical pulses permits to generate broadband frequency combs with lower average power when driving a microresonator with a pulse train whose repetition rate matches the cavity free-spectral range $[10,11]$.

In this work, we introduce a modified version of the Lugiato-Lefever equation (LLE) which accounts for a pulse train (or equivalently a frequency comb) as a pump. In order to validate the model, we have experimentally built an optical pulse generator to drive our WGM resonator. Numerical simulations based on the split-step Fourier algorithm are then performed and compared to the experimental results. One of the main focus of our work is the asymmetric broadening of the generated frequency combs when a mismatch between the pulse train repetition rate and the free-spectral range of the cavity is accounted for.

The article is structured as follows. The experimental system under study is introduced in Sec. II. Section III is devoted to the modeling of the optical pulse generation. The LLE corresponding to the WGM resonator in the pulse-pump is established in Sec. IV, while Sec. V discusses the results of the numerical simulations and experimental measurements. The final section provides some concluding remarks.

\section{EXPERIMENTAL SYSTEM}

The system under study is presented in displayed Fig. 1. It features two sub-units, namely an ultrashort optical pulse generator with tunable repetition rate, and a WGM resonator. 


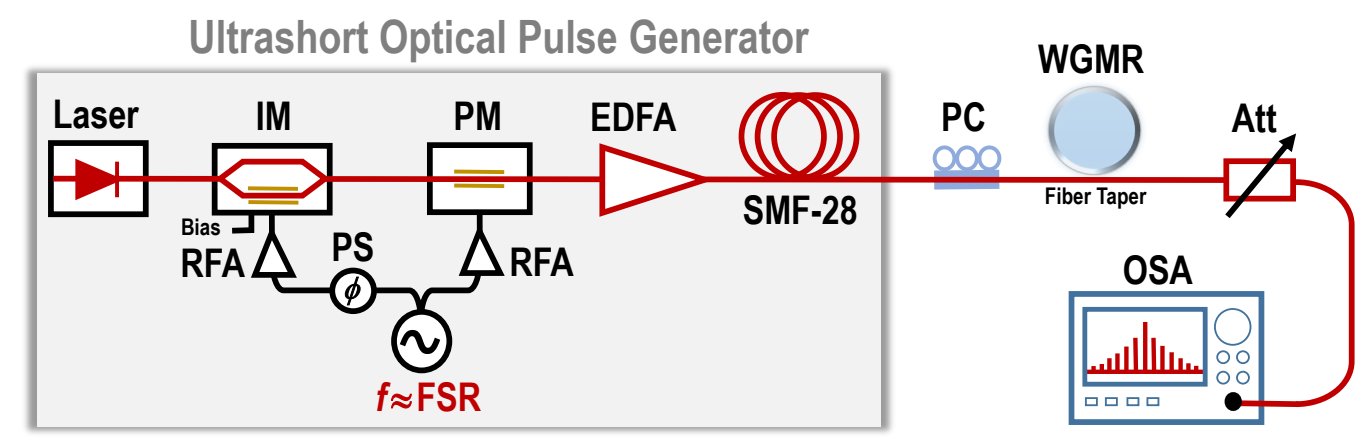

FIG. 1: (Color online) Experimental setup use to drive a WGM resonator with quasi-synchronous ultrashort optical pulses. The mm-size WGM resonator in this experiment is made of magnesium fluoride, and has an FSR of approximately 6 GHz with $Q$ in excess of $10^{9}$ at $1550 \mathrm{~nm}$. PC: Polarization controller; IM: Intensity modulator; PM: Phase modulator; EDFA: Erbium-doped fiber amplifier; RFA: Radiofrequency amplifier; PS: Phase shifter; SMF-28: Single-mode fiber spool; WGMR: WGM resonator; Att: Optical attenuator; OSA: Optical spectrum analyzer.

The optical pulse generator relies on time-lens solitonassisted compression [12], that has the capability to yield picosecond pulses with $\mathrm{GHz}$ repetition rates and wattlevel peak power. The optical source of the pulse generator is a narrow linewidth continuous-wave (CW) laser of power $P_{\mathrm{L}}=10 \mathrm{~mW}$ and wavelength $\lambda_{\mathrm{L}} \simeq 1550 \mathrm{~nm}$. An integrated Mach-Zehnder intensity modulator with half-wave voltages $V_{\pi_{\mathrm{RF}}}=4.2 \mathrm{~V}$ and $V_{\pi_{\mathrm{DC}}}=5.85 \mathrm{~V}$ is used to carve pulses out of the $\mathrm{CW}$ optical signal - the so-called pre-pulses. A phase modulator of half-voltage $V_{\pi_{\mathrm{p}}}=3.1 \mathrm{~V}$ is then used to apply a quadratic phase profile (chirp) in the time domain on the pulses, in the same fashion a lens applies a quadratic phase profile in the spatial domain to focus a light beam. Both the amplitude and phase modulators are driven by the a radiofrequency $(\mathrm{RF})$ frequency synthesizer at angular frequency $f_{\mathrm{s}}$. Two $\mathrm{RF}$ amplifiers are used to adjust the modulating signal powers to the desired levels in each path. In order to tune the RF phase difference between the input signals fed into two modulators, a phase shifter is placed on either one of the path (here, it is the amplitude modulator path). The use of a RF frequency synthesizer allows us to tune the pulse train repetition rate to any arbitrary value from $10 \mathrm{MHz}$ to several tens of $\mathrm{GHz}$ with $\mathrm{kHz}$ precision. A low noise erbium-doped fiber amplifier (EDFA) with $33 \mathrm{~dB}$ gain is used to amplify the pre-pulses in order to reach a high power before being launched into a standard SMF-28 fiber spool of length $L=3.6 \mathrm{~km}$, where they undergo time-lens soliton compression. One of the most interesting advantage of this architecture is that the maximum achievable peak power is far superior to the output power of the EDFA.

The output signal of the fiber spool is a train of ps-wide solitons with a repetition rate equal to $f_{\mathrm{S}}$. These pulses are coupled via a fiber taper into a custom-made magnesium fluoride $\left(\mathrm{MgF}_{2}\right)$ WGM resonator with an intrinsic quality factor in excess of $10^{9}$ at $1550 \mathrm{~nm}[13,14]$. The diameter of this resonator is $d \simeq 12 \mathrm{~mm}$ and its group velocity index is $n_{\mathrm{g}}=1.37$, corresponding to a free spec- tral range (or FSR) equal to $f_{\mathrm{R}}=c / \pi d n_{\mathrm{g}}=5.782 \mathrm{GHz}$, where $c$ is the velocity of light in vacuum. The bandwidth of a resonance in this resonator has been measured to be approximately $1 \mathrm{MHz}$. In order to couple the optical pulses (or frequency comb in the spectral domain) generated by the system to the WGM resonator, we need to very precisely match the repetition rate of the pulse train (i.e., the driving frequency of the frequency synthesizer) with the FSR of the cavity. In other words, we need to have $f_{\mathrm{S}} \simeq f_{\mathrm{R}}$, which is approximately $6 \mathrm{GHz}$ in our case as indicated earlier.

The output signal of the resonator is eventually attenuated before being sent to an optical spectrum analyzer that displays the output optical frequency comb.

\section{MODEL FOR THE OPTICAL PULSE GENERATOR}

The dynamics of the optical pulse generator can be tracked via the electric field

$$
E_{\mathrm{f}}(z, t)=\frac{1}{2} \mathcal{E}_{\mathrm{f}}(z, t) e^{i \omega_{\mathrm{L}} t}+\frac{1}{2} \mathcal{E}_{\mathrm{f}}^{*}(z, t) e^{-i \omega_{\mathrm{L}} t}
$$

where $\omega_{\mathrm{L}}$ is the angular frequencies associated with the $1550 \mathrm{~nm} \mathrm{CW}$ laser, and $\mathcal{E}_{\mathrm{f}}(z, t)$ is the complex-valued slowly-varying envelope of the optical field, which is normalized in such a way that the optical power is readily obtained as $\left|\mathcal{E}_{\mathrm{f}}(z, t)\right|^{2}$ in units of watts.

The output field of the $\mathrm{CW}$ laser diode (extreme left of Fig. 1) has an envelope $\mathcal{E}_{\mathrm{L}}=\sqrt{P_{\mathrm{L}}}$. This signal is then amplitude- and phase-modulated with a driving RF signal proportional to $V(t)=V_{0} \cos \Omega_{\mathrm{S}} t$ (with $\Omega_{\mathrm{S}}=2 \pi f_{\mathrm{S}}$ ) before being amplified by the EDFA. As a consequence, the slowly-varying envelope of the optical field at the input of the optical fiber is obtained as [15]

$$
\mathcal{E}_{\mathrm{f}}(0, t)=\sqrt{\kappa_{o} G_{o} P_{\mathrm{L}}}
$$




$$
\begin{aligned}
& \times \cos \left\{\frac{\pi}{2} \frac{V_{0}}{V_{\pi_{\mathrm{RF}}}} \cos \Omega_{\mathrm{S}} t+\frac{\pi}{2} \frac{V_{\mathrm{B}}}{V_{\pi_{\mathrm{DC}}}}\right\} \\
& \times \exp \left\{i \pi \frac{\eta V_{0}}{V_{\pi_{\mathrm{p}}}} \cos \Omega_{\mathrm{S}} t+\Delta \Phi\right\}
\end{aligned}
$$

where $\kappa_{o}$ stands for all the optical losses between the output of the laser and the input of the optical fiber spool, $G_{O}$ is the gain of the EDFA, $V_{\mathrm{B}}$ is the bias voltage of the intensity modulator, $\Delta \Phi$ is the phase-shift induced between phase and intensity modulations signals, while $\eta$ is a dimensionless parameter measuring the amplitude ratio between the phase and intensity electrooptic modulations (controlled by the imbalance between the RF amplifiers in the two modulation paths).

The field $\mathcal{E}_{\mathrm{f}}(0, t)$ is a train of chirped pre-pulses with quasi-sinusoidal shape, which is then launched into the fiber spool, where it evolves as $\mathcal{E}_{\mathrm{f}}(z, t)$ following the propagation equation

$$
\frac{\partial \mathcal{E}_{\mathrm{f}}}{\partial z}=-\frac{\alpha_{\mathrm{f}}}{2} \mathcal{E}_{\mathrm{f}}-\beta_{1 \mathrm{f}} \frac{\partial \mathcal{E}_{\mathrm{f}}}{\partial t}-i \frac{\beta_{2 \mathrm{f}}}{2} \frac{\partial^{2} \mathcal{E}_{\mathrm{f}}}{\partial t^{2}}+i \gamma_{\mathrm{f}}\left|\mathcal{E}_{\mathrm{f}}\right|^{2} \mathcal{E}_{\mathrm{f}} .
$$

The fiber parameters are the loss parameter $\alpha_{\mathrm{f}}=$ $0.046 \mathrm{~km}^{-1}$, the inverse group velocity $\beta_{1 \mathrm{f}}=1 / v_{\mathrm{g}, \mathrm{f}}=$ $\left[2.0 \times 10^{8} \mathrm{~m} / \mathrm{s}\right]^{-1}$, the group-velocity dispersion $\beta_{2 \mathrm{f}}=$ $-20 \mathrm{ps}^{2} / \mathrm{km}$, and the Kerr nonlinearity parameter $\gamma_{\mathrm{f}}=$ $1.1 \mathrm{~W}^{-1} / \mathrm{km}$. The above Eq. (3) is subsequently reduced to the well-known nonlinear Schrödinger equation (NLSE) with losses, by rewriting it in the moving frame that cancels the first-order term $\beta_{1 \mathrm{f}} \partial_{t} \mathcal{E}_{\mathrm{f}}$. After numerical simulation using the split-step Fourier algorithm and initial condition $\mathcal{E}_{\mathrm{f}}(0, t)$, the NLSE permits to obtain the output signal $\mathcal{E}_{\mathrm{f}}(L, t)$ of the fiber spool, which becomes the input signal for the WGM resonator.

\section{THE LUGIATO-LEFEVER EQUATION MODEL FOR A WGM RESONATOR WITH A PULSED PUMP}

The Lugiato-Lefever equation (LLE) is a well established model to describe the intracavity dynamics of laser fields in WGM resonators with Kerr nonlinearity, when pumped by a continuous-wave laser $[4,16,17]$. When the pump field is a train of quasi-synchronous optical pulses, the LLE ruling the intracavity dynamics becomes

$$
\begin{aligned}
\frac{\partial \mathcal{E}}{\partial t}= & -\kappa \mathcal{E}+i \sigma \mathcal{E}-\Omega_{\Delta} \frac{\partial \mathcal{E}}{\partial \theta}+i v_{g} \sum_{k=2}^{K}\left(i \Omega_{\mathrm{R}}\right)^{k} \frac{\beta_{k}}{k !} \frac{\partial^{k} \mathcal{E}}{\partial \theta^{k}} \\
& +i v_{g} \gamma|\mathcal{E}|^{2} \mathcal{E}+\sqrt{2 \kappa_{\mathrm{t}} / T_{\mathrm{R}}} \mathcal{E}_{\text {in }}(\theta),
\end{aligned}
$$

where $\mathcal{E}(\theta, t)=\sum_{l} \mathcal{E}_{l}(t) e^{i l \theta}$ is the envelope of the total intra-cavity field normalized such that $|\mathcal{E}|^{2}$ is the corresponding power in watts, and $\mathcal{E}_{l}(t)$ is the field envelope for the mode of reduced azimuthal order $l=\ell-\ell_{0}$. This intracavity field depends on the azimuthal angle along the closed-path circumference of the resonator $\theta \in[-\pi, \pi]$ and on the time variable $t$. The parameter

$$
\Omega_{\Delta}=\Omega_{\mathrm{S}}-\Omega_{\mathrm{R}}=2 \pi f_{\Delta}
$$

is the frequency detuning between the pulse repetition rate and the cavity free-spectral range. It should be noted that here, the retarded time-frame is characterized by the angle $\Omega_{\mathrm{S}} t$ (and not $\Omega_{\mathrm{R}} t$ like in the CW pump case), so that stationary patterns are rotating at angular frequency $\Omega_{\mathrm{S}}$ instead of $\Omega_{\mathrm{R}}$. Equivalently, the reference comb is equidistant with frequency spacing $\Omega_{\mathrm{s}}$. As a consequence, when $\Omega_{\Delta} \neq 0$, the patterns should experience a drift (or walk-off) in the cavity.

The loss parameters in this equation are the internal ("i"), coupling or external ("e"), and total coupling bandwidths $\Delta \omega_{\mathrm{i}, \mathrm{e}, \mathrm{tot}}=\omega_{0} / Q_{\mathrm{i}, \mathrm{e}, \mathrm{tot}}$, where $\omega_{0}$ is the cold-cavity eigenfrequency of the pumped mode. We can then redefine these coefficients as $\kappa_{\mathrm{i}, \mathrm{e}}=\Delta \omega_{\mathrm{i}, \mathrm{e}} / 2$, and the total (or loaded) half-linewidth $\kappa=\Delta \omega_{\text {tot }} / 2$ is now rewritten as $\kappa=\kappa_{\mathrm{i}}+\kappa_{\mathrm{e}}(\sim 2 \pi \times 1 \mathrm{MHz})$. The frequency detuning between the laser and the resonance frequency of the pumped mode is $\sigma=\omega_{\mathrm{L}}-\omega_{0}$. The intracavity round-trip time is $T_{\mathrm{S}}=2 \pi / \Omega_{\mathrm{S}}$, and the $k$-th order dispersion parameters are $\beta_{k}$. The four-wave mixing (FWM) term induces a global coupling weighted by the nonlinear parameter $\gamma=n_{2} \omega_{\mathrm{L}} / c A_{\text {eff }}$, where $n_{2}$ is the Kerr nonlinearity, and $A_{\text {eff }}=V_{\text {eff }} / \pi d$ is the effective mode area inside the resonator, with $d$ being its diameter. The parameter $v_{\mathrm{g}}=c / n_{\mathrm{g}}$ stands for group velocity in the resonator at the pump frequency. In this study, we will restrict ourselves to second-order dispersion (with $\left.\beta_{2} \sim-10 \mathrm{ps}^{2} / \mathrm{km}\right)$, and neglect higher-order dispersion $\left(\beta_{k} \equiv 0\right.$ for $\left.k \geq 3\right)$.

The input field of the WGM resonator is the output field $\mathcal{E}_{\mathrm{f}}(L, t)$ of the optical pulse generator, following

$$
\mathcal{E}_{\text {in }}(\theta) \equiv \mathcal{E}_{\mathrm{f}}(L, t)=\mathcal{E}_{\mathrm{f}}\left(L, \theta / \Omega_{\mathrm{S}}\right) .
$$

We have here accounted for the fact that $t$ is a fast time variable time in the NLSE of Eq. (3), which in our case has to be mapped to the spatial variable $\theta$ [note that $t$ in the LLE obtained in Eq. (4) is a slow time variable]. On the other hand, the output field of the WGM resonator is defined as

$$
\mathcal{E}_{\text {out }}(\theta, t)=-\mathcal{E}_{\text {in }}(\theta)+\sqrt{2 \kappa_{\mathrm{e}} T_{\mathrm{R}}} \mathcal{E}(\theta, t) .
$$

The comparison between the numerical simulations and experimental results will be carried out using the output signal $\left|\mathcal{E}_{\text {out }}\right|^{2}$, and not $|\mathcal{E}|^{2}$ which is experimentally inaccessible (except in the add-drop configuration). The LLE corresponding to the conventional case of $\mathrm{CW}$ pumping is recovered by setting $\Omega_{\Delta} \equiv 0$, and by replacing the pulsed pump $\mathcal{E}_{\text {in }}(\theta)$ by a constant field $\sqrt{P_{0}}$, where $P_{0}$ is the laser of the pump laser.

It is noteworthy that an equation similar to Eq. (4) was studied in refs. [18, 19]. It was also used in ref. [20] to investigate spontaneous breaking of the time-reversal symmetry in a passive Kerr fiber resonator driven by a 

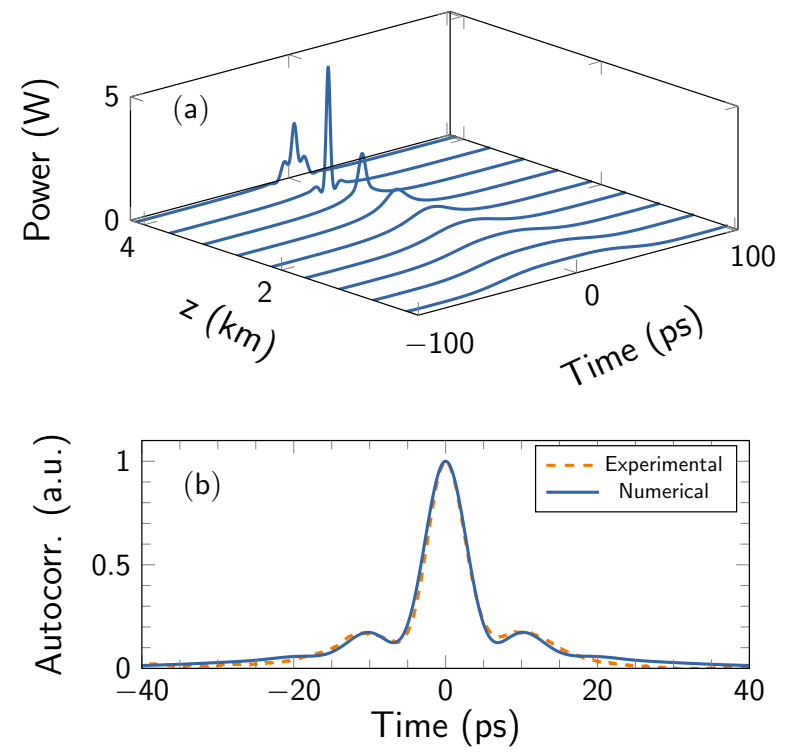

FIG. 2: (Color online) (a) Numerical simulation based on Eq. (3) for the time-lens soliton compression as the quasisinusoidal prepulses are propagating along the fiber spool. The parameters of the simulation for the initial condition in Eq. (2) are $\kappa_{o} G_{o} P_{\mathrm{L}}=420 \mathrm{~mW}, V_{0}=2 \mathrm{~V}, \Delta \Phi=0$ and $\eta=3$. (b) Comparison between the experimental and simulated autocorrelation plots for the compressed (output) optical pulses. This plot permits to evaluate the full-width at half maximum to a value around $4 \mathrm{ps}$.

synchronous pulsed pump (with no frequency mismatch). A detailed mathematical analysis of that model was later on carried out in ref. [21], where the authors analyzed the bifurcation behavior of the system and the emergence of asymmetric states as a function of the pump power.

For our numerical simulations, it is convenient to rewrite Eqs. (4) and (7) in dimensionless form. For that purpose we introduce the following dimensionless variables:

$$
\psi=\left(\gamma v_{g} / \kappa\right)^{\frac{1}{2}} \mathcal{E} \text { and } \tau=\kappa t
$$

for the intracavity field and the time, where $\kappa$ is the loaded half-linewidth.

If we restrict ourselves to second-order dispersion, the dimensionless model is now rewritten as

$$
\begin{aligned}
\frac{\partial \psi}{\partial \tau}= & -(1+i \alpha) \psi+\varepsilon \frac{\partial \psi}{\partial \theta}-i \frac{\beta}{2} \frac{\partial^{2} \psi}{\partial \theta^{2}} \\
& +i|\psi|^{2} \psi+F \mathcal{S}(\theta),
\end{aligned}
$$

where we recover the real-valued dimensionless parameters that characterize the conventional the LLE, and that stand for cavity detuning and group-velocity dispersion following

$$
\alpha=-\frac{\sigma}{\kappa} \text { and } \beta=\beta_{2} \frac{v_{g} \Omega_{\mathrm{R}}^{2}}{\kappa}
$$

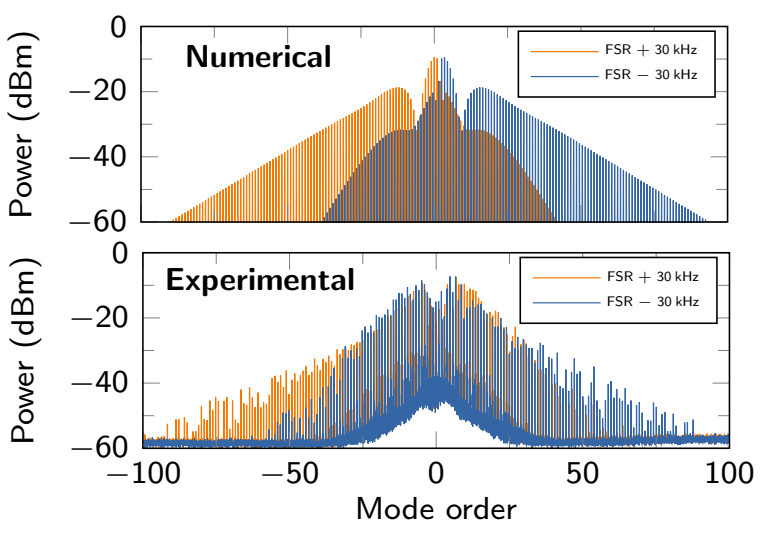

FIG. 3: (Color online) Kerr comb spectra at the output of the WGMR for a repetition rate mismatch of $\pm 30 \mathrm{kHz}$ relatively to the FSR. Top: Numerical simulation using Eq. (9) with parameter $\alpha=0, F=8, \beta=-0.005$, and considering a Gaussian pulse with a 4.2 ps width. Bottom: Experimental spectra using the experimental setup presented in Fig. 1.

The new dimensionless parameter is

$$
\varepsilon=-\frac{\Omega_{\Delta}}{\kappa},
$$

which accounts for the frequency mismatch between the pulse train repetition rate and the cavity FSR. Note that $\varepsilon$ is typically of the same order of magnitude as $\alpha$ : Resonant pumping requires $-1<\varepsilon<1$, while perfectly synchronous pumping corresponds to $\varepsilon=0$.

The normalization of the pulsed pump term is such that

$$
F=\sqrt{\frac{2 \gamma v_{g}}{T_{\mathrm{R}}} \frac{\kappa_{\mathrm{e}}}{\kappa^{3}}} \sqrt{P_{\mathrm{S}}},
$$

where

$$
\begin{aligned}
P_{\mathrm{S}} & =\frac{1}{T_{\mathrm{S}}} \int_{-T_{\mathrm{S}} / 2}^{T_{\mathrm{S}} / 2}\left|\mathcal{E}_{\mathrm{f}}(L, t)\right|^{2} d t \\
& =\frac{1}{2 \pi} \int_{-\pi}^{\pi}\left|\mathcal{E}_{\text {in }}(\theta)\right|^{2} d \theta
\end{aligned}
$$

is the average power of the optical pulse train, while

$$
\mathcal{S}(\theta)=\frac{\mathcal{E}_{\text {in }}(\theta)}{\sqrt{P_{\mathrm{S}}}}
$$

is its normalized complex-valued envelope that fulfills the condition

$$
\frac{1}{2 \pi} \int_{-\pi}^{+\pi}|\mathcal{S}(\theta)|^{2} d \theta=1
$$

We also note that the dimensionless output field is

$$
\psi_{\text {out }}=-F \mathcal{S}(\theta)+2 \rho \psi
$$




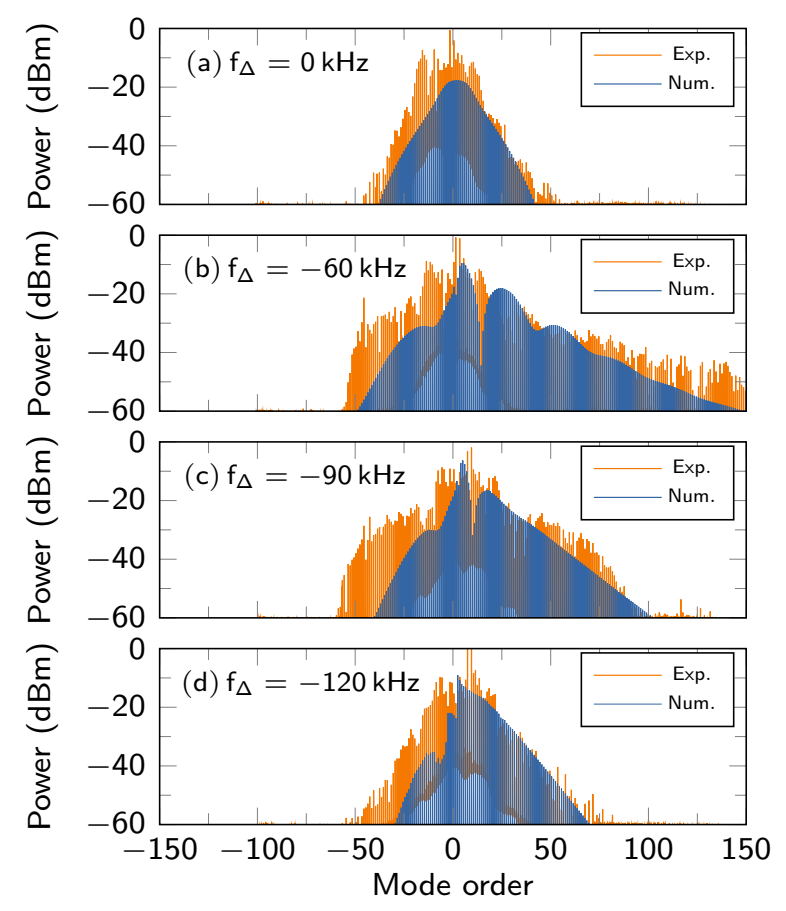

FIG. 4: (Color online) Experimental and numerical spectra for various values of the frequency mismatch $f_{\Delta}$. The parameters for the numerical simulations were $\alpha=0$ and $\beta=-0.008$. The input pulse was a Gaussian with a 4.2 ps pulsewidth.

with $\rho=\kappa_{\mathrm{e}} / \kappa$ being the ratio between out-coupling and total losses. The usual dimensionless LLE for CW pumping is recovered by setting $\varepsilon \equiv 0$ and $\mathcal{S}(\theta) \equiv 1$.

The new dynamical equation corresponding to our system is Eqs. (9), with the normalization constraint of Eq. (15). It appears that this model introduces two new features relatively to its CW counterpart, namely the frequency mismatch $\varepsilon$ and the pulse profile $\mathcal{S}(\theta)$. The latter is complex-valued and infinite-dimensional, but it is very likely that its most important characteristic will be its angular pulsewidth $w$, which controls the spectral span of the pump pulses. Therefore, in first approximation, we can consider that pulsed pumping adds two new parameters to the classical LLE, namely $\varepsilon$ and $w$.

\section{EXPERIMENTAL AND NUMERICAL RESULTS}

We have used the experimental system presented in Fig. 1 to generate the optical pulses needed to drive the WGM resonator. The autocorrelation profile show that pulses were shortened down to 4 ps, as shown in Fig. 2, and the output peak power was $1.5 \mathrm{~W}$ (corresponding to an average power of $40 \mathrm{~mW}$ ). The autocorrelation function has the characteristic structure of soliton compression associated with the Peregrine soliton profile [22].

This signal was then used to pump the WGM res-

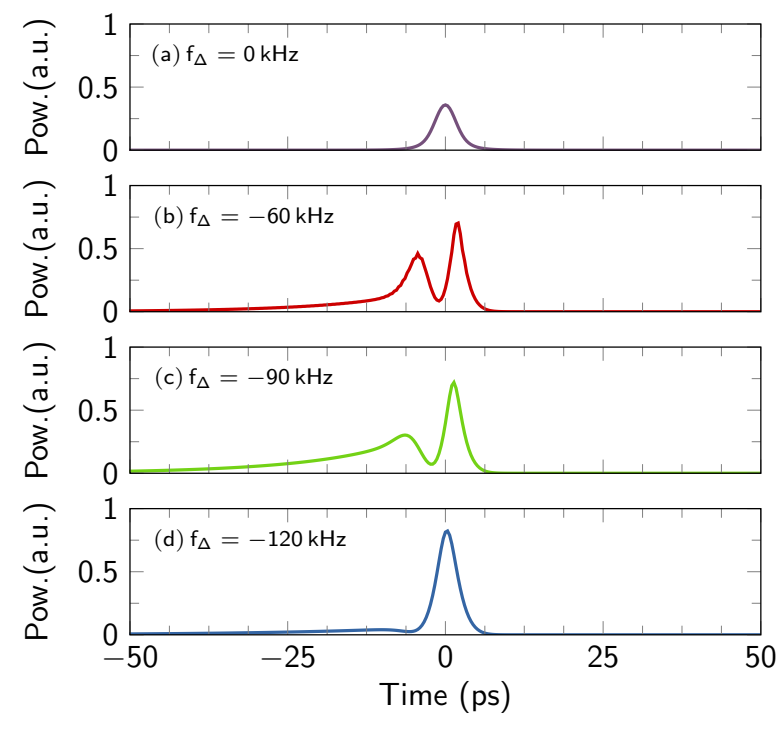

FIG. 5: (Color online) Numerical simulation showing the variation of the pulseshape for various values of the frequency mismatch $f_{\Delta}$. The parameters for the numerical simulations are the same as those in Fig. 4.

onator, and the resulting spectra are shown in Fig. 3. We have laid a particular emphasis on the role of the frequency mismatch $\Omega_{\Delta}=\Omega_{\mathrm{S}}-\Omega_{\mathrm{R}}$ between the pulse train repetition rate and the resonator FSR. We observe that the spectra undergo asymmetric broadening as the driving signal repetition rate is mismatched, and that the direction of the asymmetry is directly correlated with the sign of the mismatch.

This phenomenon can be intuitively understood when we picture the position of the frequency comb used to drive the resonator relative to the position of each resonance. When the repetition rate of the pulse train is superior to the FSR of the cavity, part of the spectrum that is broadened (high-frequency part of the optical spectrum) corresponds to the spectral area of the frequency comb which is red-detuned in the resonances; conversely, the spectral area of the frequency comb on the bluedetuned side does not undergo any broadening. When the repetition rate is inferior to the FSR, the opposite phenomenology is observed. This dynamical behavior is reminiscent of the mechanism needed to generate dissipative Kerr solitons with microresonators [23], where a scan in the red detuned side of the resonance was necessary to generate broadband frequency combs.

In order to reach a deeper understanding of the phenomenon, the model introduced in Eq. (9) has been numerically implemented, using the split-step Fourier algorithm. The simulation displays a phenomenon similar to the one observed experimentally. When $\varepsilon$ is negative, the repetition rate of the pulse train is higher than the FSR of the cavity and the spectrum broadens toward the lower frequencies. On the other hand, when $\varepsilon$ is positive, 
the spectrum shifts toward the higher frequencies. We observe a slope of about approximately $0.5 \mathrm{~dB}$ per mode on both the experimental and numerical spectra, leading us to the conclusion that our model is in good qualitative agreement with the experiments. We can rule out Raman self-pumping as the key effect behind the phenomenon we observed, because the the related self-frequency shift would continuously shifts the soliton pulse toward lower frequencies. However, in our experiment, we were able to observe both redshift and blue shifts.

We have also investigated the effect on the frequency mismatch $\Omega_{\Delta}$ between the repetition rate of the pulses and FSR of the cavity on the dynamics of the system. Figure 4 shows the experimental and numerical spectra for four different detuning ranging from 0 to $-120 \mathrm{kHz}$, while Fig. 5 displays the corresponding numerical pulse shapes. When the detuning is null, we observe minimal spectrum broadening, and the pulses remain Gaussian. As $f_{\Delta}$ is set to $-60 \mathrm{kHz}$, we observe a large asymmetrical broadening, tilted towards higher frequencies. This broadening corresponds to a pulse doubling (or splitting) in the time domain. When the detuning is decreased to $-90 \mathrm{kHz}$, the bandwidth broadening is reduced, thereby indicating that the broadening process has a nontrivial dependence with $\Omega_{\Delta}$. Accordingly, the pulse splitting in the time domain is gradually reversed. Further decrease of the detuning to $-120 \mathrm{kHz}$ leads to a the spectrum that is very similar to one obtained with resonant pumping $\left(f_{\Delta}=0 \mathrm{kHz}\right)$, with the time-domain pulse displaying a single but slightly asymmetric peak. Both the experimental measurements and the numerical simulations are in agreement while describing this phenomenology, which confirms the notable effect of frequency mismatch on the bandwidth of the output comb spectra. One should note however that we have pumped two different mode families in Figs. 3 and 4, thereby explaining why two different values of dispersion were used in the numerical simulations. One can also note that the envelope of the spectra are not smooth, and this can be attributed to instability within the resonator (thermal instabilities, jitter of the source).

Another interesting point is the small frequency range around which the system exhibits asymmetric broadening. Both the experimental measurements and the numerical simulations indicate that this range is roughly about $100 \mathrm{kHz}$ in our case, even though the resonance on which we are locked is approximately $1 \mathrm{MHz}$ wide. Our interpretation of this phenomenon is that when we set a detuning of $\Omega_{\Delta}$, only the the first pair of teeth of the frequency comb we use to pump the resonator is detuned by that amount relative to the resonance, as the the $n$ th pair of comb teeth is detuned by $n \Omega_{\Delta}$. This means that as the detuning is increased, the sidemodes of the pump comb are increasingly becoming out-of-resonance input signals, and their influence in the intracavity dy- namics thereby decreases. Asymptotically, only the central frequency of the pump comb becomes relevant when $\Omega_{\Delta} \gg \kappa$ and the resulting comb becomes in all points similar to the one obtained by a continuous-wave pump.

\section{CONCLUSION}

We have introduced a new version of the LugiatoLefever equation which accounts for the use of a pulse train as the quasi-synchronous driving field of a WGM resonator. We have presented the experimental setup used to drive the WGMR with pulses, as well as the corresponding theoretical model. The numerical simulations and experimental measurements have shown the phenomenon of asymmetric broadening of frequency comb when a mismatch between the cavity FSR and the repetition rate of the pulse train was induced.

This work could be extended in numerous ways in the near future. From a mathematical viewpoint, it is necessary to perform a full bifurcation analysis based on the normal form theory in order to understand the nature and stability of the patterns that can emerge in the system [24-27]. The consideration of other nonlinear [28, 29] or thermal [30-32] effects would lead to additional terms that are relevant in order to provide a more accurate description of the intracavity dynamics. From the applications perspective, this system and the associated model would permit to optimize self-oscillators incorporating WGM oscillators in their feedback loop [33, 34].

One of the most important application of our work belong to the realm of quantum optics. Indeed, quantum frequency combs are the ideal technological paradigm for the creation and manipulation of frequency bin states [35-40]. High- $Q$ WGM resonators with pulsed pumping arose in recent years as ideal systems from that perspective as they have the potential to provide a chipscale platform for the next generation of quantum photonic systems, as it enables time bin protocols and drastically reduces the optical power needed to drive the resonators [41-47]. We expect that a quantization of the classical equations would allow us to gain a deeper understanding of these systems.

\section{Acknowledgment}

The authors acknowledge useful discussions with Mariana Haragus. They also acknowledge support from the Agence Nationale de la Recherche (I-SITE BFC: ANR-15-IDEX-0003; EIPHI Graduate School: ANR-17EURE-0002). 
[1] P. DelHaye, A. Schliesser, O. Arcizet, T. Wilken, R. Holzwarth, and T. J. Kippenberg, Nature 450, 1214 (2007).

[2] D. V. Strekalov, C. Marquardt, A. B. Matsko, H. G. Schwefel, and G. Leuchs, J. Opt. 18, 123002 (2016).

[3] T. J. Kippenberg, A. L. Gaeta, M. Lipson, and M. L. Gorodetsky, Science 361 (2018).

[4] A. Pasquazi, M. Peccianti, L. Razzari, D. J. Moss, S. Coen, M. Erkintalo, Y. K. Chembo, T. Hansson, S. Wabnitz, P. DelHaye, et al., Phys. Rep. 729, 1 (2018).

[5] D. V. Strekalov and N. Yu, Phys. Rev. A 79, 041805 (2009).

[6] T. Hansson and S. Wabnitz, Phys. Rev. A 90, 013811 (2014).

[7] Y. Okawachi, M. Yu, K. Luke, D. O. Carvalho, S. Ramelow, A. Farsi, M. Lipson, and A. L. Gaeta, Opt. Lett. 40, 5267 (2015).

[8] W. Wang, S. T. Chu, B. E. Little, A. Pasquazi, Y. Wang, L. Wang, W. Zhang, L. Wang, X. Hu, G. Wang, et al., Sci. Rep. 6, 28501 (2016).

[9] C. Bao, P. Liao, A. Kordts, L. Zhang, M. Karpov, M. H. Pfeiffer, Y. Cao, Y. Yan, A. Almaiman, G. Xie, et al., Opt. Lett. 42, 595 (2017).

[10] E. Obrzud, S. Lecomte, and T. Herr, Nat. Photon. 11, 600 (2017).

[11] M. Anderson, R. Bouchand, E. Obrzud, J. Liu, S. Karlen, S. Lecomte, T. Herr, and T. J. Kippenberg, in CLEO: Science and Innovations (Optical Society of America, 2019), pp. STu3J-3.

[12] M. Hanna, P.-A. Lacourt, S. Poinsot, and J. M. Dudley, Opt. Express 13, 1743 (2005).

[13] A. Coillet, R. Henriet, K. P. Huy, M. Jacquot, L. Furfaro, I. Balakireva, L. Larger, and Y. K. Chembo, J. Vis. Exp. p. e50423 (2013).

[14] G. Lin and Y. K. Chembo, Opt. Mater. X 2, 100017 (2019).

[15] Y. K. Chembo, A. Hmima, P.-A. Lacourt, L. Larger, and J. M. Dudley, J. Lightw. Technol. 27, 5160 (2009).

[16] Y. Chembo, D. Gomila, M. Tlidi, and C. R. Menyuk, Eur. Phys. J. D 71 (2017).

[17] L. Lugiato, F. Prati, M. Gorodetsky, and T. Kippenberg, Phil. Trans. Roy. Soc. A 376, 20180113 (2018).

[18] M. Haelterman, S. Trillo, and S. Wabnitz, Opt. Commun. 91, 401 (1992).

[19] P. Parra-Rivas, D. Gomila, M. A. Matias, P. Colet, and L. Gelens, Opt. Express 22, 30943 (2014).

[20] Y. Xu and S. Coen, Opt. Lett. 39, 3492 (2014).

[21] J. Rossi, R. Carretero-González, P. Kevrekidis, and M. Haragus, J. Phys. A 49, 455201 (2016).

[22] A. Tikan, C. Billet, G. El, A. Tovbis, M. Bertola, T. Sylvestre, F. Gustave, S. Randoux, G. Genty, P. Suret, et al., Phys. Rev. Lett. 119, 033901 (2017).

[23] T. Herr, V. Brasch, J. D. Jost, C. Y. Wang, N. M. Kondratiev, M. L. Gorodetsky, and T. J. Kippenberg, Nat. Photon. 8, 145 (2014).

[24] T. Miyaji, I. Ohnishi, and Y. Tsutsumi, Phys. D 239,
2066 (2010).

[25] G. Kozyreff, Phys. D 241, 939 (2012).

[26] C. Godey, Eur. Phys. J. D 71, 131 (2017).

[27] L. Delcey and M. Haragus, Phil. Trans. Roy. Soc. A 376 , 20170188 (2018).

[28] G. Lin, S. Diallo, J. M. Dudley, and Y. K. Chembo, Opt. Express 24, 14880 (2016).

[29] G. Lin and Y. K. Chembo, Opt. Lett. 41, 3718 (2016).

[30] S. Diallo, G. Lin, and Y. K. Chembo, Opt. Lett. 40, 3834 (2015)

[31] K. Saleh and Y. K. Chembo, Opt. Express 24, 25043 (2016).

[32] J. R. Stone, T. C. Briles, T. E. Drake, D. T. Spencer, D. R. Carlson, S. A. Diddams, and S. B. Papp, Phys. Rev. Lett. 121, 063902 (2018).

[33] M. Peccianti, A. Pasquazi, Y. Park, B. E. Little, S. T. Chu, D. J. Moss, and R. Morandotti, Nat. Commun. 3, 1 (2012).

[34] K. Saleh, G. Lin, and Y. K. Chembo, IEEE Photon. J. 7, 1 (2014).

[35] M. Bloch, S. W. McLaughlin, J.-M. Merolla, and F. Patois, Opt. Lett. 32, 301 (2007).

[36] L. Olislager, J. Cussey, A. T. Nguyen, P. Emplit, S. Massar, J.-M. Merolla, and K. P. Huy, Phys. Rev. A 82, 013804 (2010).

[37] L. Olislager, I. Mbodji, E. Woodhead, J. Cussey, L. Furfaro, P. Emplit, S. Massar, K. P. Huy, and J.-M. Merolla, New J. Phys. 14, 043015 (2012).

[38] L. Olislager, E. Woodhead, K. P. Huy, J.-M. Merolla, P. Emplit, and S. Massar, Phys. Rev. A 89, 052323 (2014).

[39] B. Galmès, K. Phan-Huy, L. Furfaro, Y. K. Chembo, and J.-M. Merolla, Phys. Rev. A 99, 033805 (2019).

[40] N. B. Lingaraju, H.-H. Lu, S. Seshadri, P. Imany, D. E. Leaird, J. M. Lukens, and A. M. Weiner, Opt. Express 27, 38683 (2019).

[41] Y. K. Chembo, Phys. Rev. A 93, 033820 (2016).

[42] C. Reimer, M. Kues, P. Roztocki, B. Wetzel, F. Grazioso, B. E. Little, S. T. Chu, T. Johnston, Y. Bromberg, L. Caspani, et al., Science 351, 1176 (2016).

[43] L. Caspani, C. Reimer, M. Kues, P. Roztocki, M. Clerici, B. Wetzel, Y. Jestin, M. Ferrera, M. Peccianti, A. Pasquazi, et al., Nanophotonics 5, 351 (2016).

[44] J. A. Jaramillo-Villegas, P. Imany, O. D. Odele, D. E. Leaird, Z.-Y. Ou, M. Qi, and A. M. Weiner, Optica 4, 655 (2017).

[45] M. Kues, C. Reimer, P. Roztocki, L. R. Cortés, S. Sciara, B. Wetzel, Y. Zhang, A. Cino, S. T. Chu, B. E. Little, et al., Nature 546, 622 (2017).

[46] M. Kues, C. Reimer, J. M. Lukens, W. J. Munro, A. M. Weiner, D. J. Moss, and R. Morandotti, Nat. Photon. 13, 170 (2019).

[47] X. Lu, Q. Li, D. A. Westly, G. Moille, A. Singh, V. Anant, and K. Srinivasan, Nat. Phys. 15, 373 (2019). 